\title{
Steam-Assisted Chemical Vapor Deposition of Zeolitic Imidazolate
}

\section{Framework}

Jing-Kai Huang, ${ }^{\dagger}$ Noboru Saito, ${ }^{9} \ddagger$ Yichen Cai, ${ }^{\S}$ Yi Wan, ${ }^{\S}$ Chia-Chin Cheng, ${ }^{\S}$ Mengliu Li, ${ }^{\S}$ Junjie Shi, ${ }^{\dagger}$ Kaoru Tamada, ${ }^{\Uparrow}$ Vincent C. Tung, ${ }^{\S}$ Sean $\mathrm{Li}^{\dagger *}$ and Lain-Jong Li ${ }^{\dagger * *}$

$\dagger$ School of Materials Science and Engineering, University of New South Wales, NSW 2052, Australia.

" Institute for Materials Chemistry and Engineering (IMCE), Kyushu University, 744 Motooka, Nishi-ku, Fukuoka, 819-0395, Japan.

$\S$ Physical Sciences and Engineering Division, King Abdullah University of Science and Technology (KAUST), Thuwal 23955-6900, Saudi Arabia.

\# Department of Electronic Engineering, and Green Technology Research Center, Chang-Gung University, Taoyuan 333, Taiwan

* To whom correspondence should be addressed: (L.-J. Li) l.li@unsw.edu.au; (S. Li) sean.li@unsw.edu.au

\$ These authors contributed equally to this work.

\section{ABSTRACT:}

Direct growth of metal-organic frameworks (MOFs) on substrates is a prerequisite to incorporating them into functional platforms and microdevices. Nevertheless, available reports mostly rely on the solvent-based routes, typically altered from processes for powder synthesis which are obstacles to the nanofabrication. Besides, although few vapor-phase growth approaches were presented, the proposed procedures required multiple steps such as matrix deposition and post-conversion to obtain desired MOF films on substrates. Here, we demonstrate a steam-assisted chemical vapor deposition (CVD) method to directly synthesize highly crystalline ZIF-67 thin films at the temperature $<125^{\circ} \mathrm{C}$. With a slow deposition rate, the ZIF-67 forms a highly oriented thin film on a c-plane sapphire substrate, indicating the growth is epitaxial. Furthermore, we demonstrate the integration of directly grown CVD ZIF-67 as the active material of chemiresistors onto microelectronic chips. The ZIF-67 chemiresistors exhibit responses to the gas molecules which are capable of 
diffusing into the cage of ZIF-67 at room temperature. The proposed synthesis method of ZIF-67 thin films is simple, scalable, cost-effective, and promising for numerous applications.

Metal-organic frameworks (MOFs), a class of porous crystalline coordination polymers, are composed of metal ions joined by organic linkers to form one-, two-, or threedimensional structures. ${ }^{1-3}$ Owing to unique physical properties by designing modular construction of metal nodes and organic ligands, MOFs show the potential to enable technologies in a variety of fields including sensing, ${ }^{4,5}$ catalysis, ${ }^{6,7}$ and molecular sieving. ${ }^{8-10}$ Apart from these application fields, where MOFs are typically obtained as powder forms via established bulk synthetic approaches, more recent direction gradually targets the integration of MOFs into microelectronics, for instance, as active detecting layers, tunable conductors, low-k dielectrics and phosphor coatings. ${ }^{11}$ To further leverage the potential of MOFs in future electronic devices, the prerequisite is developing robust thin film deposition methods. To date, most reported processes for MOF deposition involve diverse solution environments such as liquid-phase epitaxy, ${ }^{12,13}$ spin coating, ${ }^{14,15}$ and drop-casting, ${ }^{4}$ which are obstacles to microelectronic fabricating process owing to the difficulty of assembling MOFs on desired locations on substrates. Also, the solution may potentially lead to chemical contamination, corrosion, and pattern deformation. In contrast, gas-phase depositing technologies are highly compatible with established microfabrication. ${ }^{16}$ Ameloot and co-workers illustrated the first strategy based on the gasphase deposition. ${ }^{17}$ They adopted a combination of atomic layer deposition (ALD) and vapor-assisted conversion (VAC) to demonstrate the transformation of conformal ALD metal oxides into crystalline MOFs. Afterward, few groups reported the use of the ALD technique to grow amorphous metal-organic hybrid thin films and subsequently crystallize via post-heat treatments. ${ }^{18,19}$ The aforementioned works mostly involve twosteps synthesis, the growth of precedent layers and post-converting treatments, which are time-consuming and incapable of in-situ controlling orientation, grain size, and surface morphologies. Besides, the ALD approach is limited to the carboxylic acid and analogue ligands likely due to the challenge of delivering other precursors in gas phases. Last, relatively high cost and slow deposition rate of ALD technology are not favorable for 
cost-effective fabrication. ${ }^{20}$ Here, we report a steam-assisted chemical vapor deposition (CVD) approach for directly producing ZIF-67 and associated zeolitic imidazolate frameworks (ZIFs) at the temperature ranging from $95^{\circ} \mathrm{C}$ to $125^{\circ} \mathrm{C}$. The introduction of steam, as a mediator, thoroughly prompts the ZIF crystal ripening. Also, the straightforward deposition method enables the growth of highly oriented ZIF thin films by properly adjusting the growth parameters. Meanwhile, we prove that the steamassisted CVD is a microelectronics-compatible route by demonstrating the fabrication of miniature ZIF-67 chemiresistors based on as-grown ZIF-67 films on chips using conventional microfabrication processes. The ZIF-67 chemiresistors exhibit response to the molecules with hydrogen bonding in ambient conditions. The corresponding sensing mechanism is elaborated as well. The proposed steam-assisted CVD approach not only opens new avenues to explore various MOFs but also expedite the integration of MOF materials in microelectronics for related applications.

We select the commercially available cobalt(II) acetylacetonate $\left[\mathrm{Co}(\mathrm{acac})_{2}\right]$ and 2methylimidazole (2-MIM) serving as the metal precursor and ligand (Figure 1a) to demonstrate the growth of ZIF-67 on substrates. Compared to other metal sources, Co(acac $)_{2}$ can be easily vaporized at $55 \sim 130^{\circ} \mathrm{C}$, and it is thermally stable, which allows the steady supply to the CVD reaction zone. ${ }^{21}$ Figure $1 \mathrm{~b}$ depicts the synthesis route of ZIF-67, which involves the coordination of deprotonated 2-MIM with $\mathrm{Co}^{2+}$, based on the nets of CoN4 tetrahedra (Figure 1c). ${ }^{22}$ Figure 1d and Figure S1 schematically illustrate the gaseous deposition process and experimental setup for growing ZIF-67 thin film by the steam-assisted reaction of $\mathrm{Co}(\mathrm{acac})_{2}$ and 2-MIM in a hot-wall CVD chamber (More details are elaborated in Experimental Section, Supporting Information). The as-grown ZIF-67 thin film on c-sapphire substrates is purple, as shown in the round inset of Figure 1d. The scanning electron microscopy (SEM) images in Figure $2 \mathrm{a}$ and $2 \mathrm{~b}$ reveal the highly crystalline morphology of the as-deposited ZIF-67 crystals, which were resulted from the low-growth rate deposition. Through extending the deposition time or applying repeated growth, the separate islands gradually merge into a uniform and high-surface-coverage layer as shown in Figure $2 \mathrm{c}$ and $2 \mathrm{e}$. On the contrary, the high-growth rate could result in a dense but rugged ZIF-67 thin film (Figure $2 \mathrm{~d}$ and $2 \mathrm{f}$ ). The films obtained by our CVD method were studied with X-ray diffraction (XRD) which clearly indicates the formation of highly crystalline ZIF-67 with 
orientation control depending on the growth rate (Figure 3a). ${ }^{4,22}$ Interestingly, compared to the diffraction pattern of the randomly oriented sample (red, high-growth rate), the sample was obtained under a low-growth rate (Figure 2e, blue) reveals only predominant (002), (004) and (006) peaks (Figure S2), which represents a high degree of [001] crystallite orientation alignment normal to the c-sapphire surface. ${ }^{13}$ In addition, the in-plane XRD pattern (Figure S3) shows only (110) and (200) peaks, which supports the proposed orientation alignment. ${ }^{23}$ These observations agree well with the above SEM observations, and the results suggest that the slow deposition of ZIF-67 on sapphire exhibits the epitaxial nature.

To further confirm the formation of ZIF-67 as illustrated in Figure 1b, Fourier-transform infrared spectroscopy (FTIR) was performed. The absorption peak at $431 \mathrm{~cm}^{-1}$ in Figure $3 \mathrm{~b}$ is attributed to the $\mathrm{Co}-\mathrm{N}$ stretching, which proves that cobalt(II) ions form coordination bonds with 2MIM. ${ }^{24}$ The resonance between the $\mathrm{N}-\mathrm{H}$ stretching mode and the $\mathrm{N}-\mathrm{H} \cdots \mathrm{N}$ "out-of-plane" bending mode at $184 \mathrm{~cm}^{-1}$ completely disappears in the spectrum of the ZIF-67 product, which suggests the presence of the linker in its deprotonated 2-MIM form, corresponding to its incorporation in the ZIF-67 framework. ${ }^{24}$ Meanwhile, the intensity of the $\mathrm{C}=\mathrm{N}$ stretching mode at $1588 \mathrm{~cm}^{-1}$ in 2-MIM is significantly suppressed, further proving the existence of the $\mathrm{Co}-\mathrm{N}$ bond. ${ }^{25}$ Also, X-ray photoelectron spectroscopy (XPS) provides the bonding information of asgrown ZIF-67 (Figure S4). The Co 2p 3/2 peaks can be extracted into two constituents, the peak of Co- $\mathrm{N}_{\mathrm{x}}$ at $781.2 \mathrm{eV}$ and its satellite peaks. ${ }^{26} \mathrm{In} \mathrm{N}$ 1s peak, the dominance of non-protonated nitrogen peak $(\sim 97 \%)$ suggests that the pyrrole nitrogen $(-\mathrm{NH}-)$ in 2-MIM has been deprotonated and connected to cobalt. ${ }^{9}$ These results suggest that the ZIF-67 thin film is successfully obtained by the steam-assisted CVD approach. Likewise, several other MOF materials such as zinc(II) 2-methylimidazolate or benzimidazolate can also be grown with the proposed growth approach (Figure S5 and S6), which demonstrates the general applicability of this steam-assisted CVD method.

Control over the MOF crystal architecture in films is crucial for expanding the scope of their application. For example, the oriented thin film provides aligned accessible pores to enhance selectivity in the separation technology ${ }^{13}$ or serve as on-chip molecular confining templates for 
precise molecular engineering. ${ }^{27}$ On the other hand, the irregular thin film morphology has a larger surface area to absorb targeted species ${ }^{28}$ or increase reacting sites. ${ }^{29}$ The competitive advantage of direct vapor deposition is that via adjusting the precursor supplying rate and reaction temperature, we can directly alter the rate of nucleation and crystal growth which practically affect the orientation of the MOF crystals. ${ }^{13}$ It is noteworthy that preceding vaporgrowth schemes involve the post-conversion of metallic matrixes to crystalline MOFs, ${ }^{17-19}$ which may not lead to epitaxial growth for crystal orientation allignment. Besides, although parts of the solution-based approach have achieved crystal geometry control, these works require either substrate-modification or participation of specific modulators in the processes to make nuclei able to locate at energy favorable sites. ${ }^{13,30}$

Notably, the presence of water steam plays an essential role in the reaction. Without water steam, the product was merely an indigo-colored coating covering on the substrate (Figure S7a and $\mathrm{S} 7 \mathrm{~b}$ ). The XRD analysis confirms that the indigo coating lacks apparent crystallinity (Figure S7c). However, attenuated total reflection Fourier-transform infrared spectroscopy (ATR-FTIR) shows that both samples (grown with and without water steam) present similar patterns in the fingerprint region (Figure S7d), which indicates the indigo-colored coating contains resembled chemical bonds with ZIF-67. ${ }^{24}$ Furthermore, two additional bands can be identified in the spectra of the indigo-colored coating. $1517 \mathrm{~cm}^{-1}$ represents the combination of $50 \% \mathrm{C}=\mathrm{O}$ stretching and $50 \% \mathrm{C}-\mathrm{H}$ in-plane bending modes, and the small absorption peak at $931 \mathrm{~cm}^{-1}$ is assigned to the coupling of $\mathrm{C}-\mathrm{O}$ stretching and $\mathrm{C}-\mathrm{CH}_{3}$ stretching. ${ }^{31}$ Each band is predominant absorption peak of metal acetylacetonate, ${ }^{31}$ which further suggests fractional acetylacetonate anions still chelate with cobalt atoms in the indigo-colored coating. Hence, the composite resulting from the condition without water vapor supply is an amorphous coordination complex. To further confirm the role of steam to the ZIF-67 formation, we selected another two solvents (methanol and ethanol) commonly used to synthesize ZIF-67 in solutions, for comparison. ${ }^{32}$ We kept the growth conditions including temperature, gas flow, steam pressure, and reaction time, the same as those used with steam. Interestingly, the samples grown under methanol or ethanol steam show similar indigo-color as mentioned above (Figure S8a). Through optical microscopy, we merely observed a few crystals formed on the substrate surface (Figure S8b). Moreover, the ZIF-67 characteristic peak in X-ray diffraction patterns is relatively weaker for the methanol and ethanol cases (Figure 
S8c). The yields of ZIF-67 in either methanol or ethanol steam were much lower than the yields in the water steam. Consequently, the above observations make us infer that the solvent steam should be capable of directing the structure formation of ZIF-67; i.e., the solvents render molecules such as ligands and metal ions mobile to enable crystal ripening. ${ }^{17}$ Also, water steam may further facilitate the deprotonation of the ligands as well as the hydration of the metal precursors to provide kinetically accessible pathways for the ZIF-67 growth. ${ }^{33}$

As the typical porous materials, ZIF-67 shows a high Langmuir surface area of $1832.2 \mathrm{~m}^{2} \mathrm{~g}^{-1}$; also, it exhibits high ambient stability and a relatively low bandgap $(1.98 \mathrm{eV})$ which provides certain electrical conductivity. ${ }^{4}$ Hence, with the capability of direct growing ZIF-67, we chose ZIF-67 as the active material to demonstrate a chemiresistor using typical microfabrication processes (as shown in Figure 4a). After the patterning of interdigital metal electrodes, the active region of the chemiresistor was further defined by photolithography. Afterward, the ZIF-67 is deposited by the steam-assisted CVD approach. The final lift-off the photoresists defines the sensing active area with ZIF-67 on it. Note that to obtain a larger surface area for perceiving analyte, deposition in a high-growth rate was adopted for achieving randomly oriented ZIF-67 thin films. Figure $4 \mathrm{~b}$ and $4 \mathrm{c}$ show the optical image of interdigital electrodes before/after the depositing process. In clear contrast, conventional approaches to prepare ZIF-based sensor devices should require the preparation of ZIF powders in solutions ahead and then the deposition of ZIF crystals on electrodes by either drop-casting or spin-coating of the MOF suspension, which results in poor crystal-crystal contact and crystal-substrate adhesion. ${ }^{11}$ Moreover, as further scaling down the device dimension or depositing on the high-aspect-ratio surface, solution casting methods shall be hampered by the capillary effect. ${ }^{17}$ As a result, compared with the conventional solution-phase methods, vapor CVD synthesis provides a more elaborate and efficient route to integrate MOF materials into compact electronic devices.

To investigate the actual performances of the ZIF-67 chemiresistor, we first measure the output characteristics of the sensor device in the environments with various relative humidities (R.H.). As specified in Figure 5a, the output current gradually increases with the R.H. value in the testing chamber. Furthermore, the output current curve (applied voltage $=10 \mathrm{~V}$ ) of the sensor shows a proper exponential relationship to the R.H. value (Figure 5b). This phenomenon should 
result from water-mediated proton conducting, i.e., Grotthuss mechanism; specifically, the absorbed water molecules develop hydrogen-bonding networks that serve as proton conduction pathways (Figure 5c). ${ }^{34}$ Hence, the higher ambient R.H. indicates that more proton conduction pathways are created in the structure of the framework, leading to higher conductance. Note that the electrical output characteristics of the indigo-colored layer were performed as well. However, due to the amorphous nature, it shows no obvious conductance in our detection limitation. In addition to moisture, the ZIF-67 chemiresistor shows various current responses to several selected solvent vapors and gases (Figure S9). Figure 5c summarizes the corresponding sensitivity of tested gases individually. Note that the sensitivity in this work is defined as Sensitivity $=\log (G t / G a)$, where $G_{t}$ and $G_{a}$ are the electrical conductance of the chemiresistor in the tested gas and the reference ambient air $\left(25^{\circ} \mathrm{C}, 1 \mathrm{~atm}, 45 \%\right.$ R.H., $\left.G_{a}=1.08 \times 10^{-10} \mathrm{~S}\right)$, respectively. It is noticeable that the sensor shows similar responses when water $\left(\mathrm{H}_{2} \mathrm{O}\right)$, methanol $(\mathrm{MeOH})$, and ethanol $(\mathrm{EtOH})$ vapors are supplied because these vapors are capable of promoting the conductance of the ZIF-67 via developing hydrogen-bonding networks in the framework. In contrast, other tested gases, which are lacking the ability to form hydrogen-bond, cause declines in the electrical conductance since these gas molecules replace indigenous moisture residing in the framework. However, isopropyl alcohol (IPA) exhibits unexpected responses during the test. Although IPA molecules indeed have the capacity for forming hydrogen-bond, the large kinetic diameter $\left(d_{k}\right)$ of IPA (4.9 $\AA$ ) might limit its diffusivity into the cage of ZIF-67 (pore size: $11.6 \AA$, pore aperture: $3.4 \AA){ }^{22,35}$ Instead, molecules of the carrier gas (nitrogen, $d_{k}=3.6 \AA$ ) can penetrate the cage of ZIF-67 with ease and then break the proton conduction pathways. ${ }^{36}$ Also, the clear difference of the sensitivity between methanol $\left(d_{k}=3.6 \AA\right)$ and ethanol $\left(d_{k}=4.5 \AA\right)$ does suggest the presence of size-dependent diffusivity. ${ }^{37}$ Figure S10a shows the typical dynamic response of the ZIF-67 sensor to different concentrations of methanol. Notably, the conductance recovery time is relatively slow when applying high methanol concentrations. This phenomenon might be ascribed to the reason that the measurement was conducted at room temperature since the gas desorption rate is positively correlated with temperature. ${ }^{38}$ The minimum concentration of analytes was adopted in the gas sensing measurement is 100 ppm, and the ZIF-67 sensor did show a certain response (Figure $\mathrm{S} 10 \mathrm{~b})$. 
In summary, a synthetic approach of common MOFs in vapor phase CVD has been achieved through the proposed steam-assisted method. It is concluded that the involvement of water steam plays a decisive role in triggering the vapor-phase reaction. By altering the rate of crystal growing and nucleation, the orientability of ZIF crystals can be realized. Additionally, a fully vapor-phase reaction environment and comparatively low-temperature growth conditions make this process highly compatible with welldeveloped microfabrication in particularly the backend of line processes. Compared with recently emerged vapor-assisted conversion fashions, the proposed steam-assisted CVD approach paces further steps in the processing of MOF materials. We believe that steamassisted CVD could be further extended to a broad range of MOF through matching precursors and steam mediators. This work represents a visible nexus between the preparation of MOF thin films and advanced engineering that has brightening prospects to realize MOF-based microelectronics.

\section{ASSOCIATED CONTENT}

The Supporting Information is available free of charge on the ACS Publications website. Instrument setup, detailed synthetic procedures, additional characterization data, and additional experiment results.

\section{AUTHOR INFORMATION}

\section{Corresponding Author}

(L.-J. Li) l.li@unsw.edu.au; (S. Li)sean.li@unsw.edu.au

\section{Author contributions}

J.-K. H. and L.-J. L. conceived the concept and experiments. J.-K. H. and N. S. carried out most of the experiments and analyses. Y. W., M. L. and J. S. performed SEM. N. S. and C.-C. C. prepared the specimens. Y. C. performed gas sensing measurements. K. T., V. T. and S. L. provided helpful opinions and suggestions. J.-K. H. and L.-J. L. wrote the manuscript. All coauthors discussed the results and commented on the manuscript at all stages. J.-K. H. and N. S. contributed equally to this work. 


\section{ACKNOWLEDGMENTS}

The authors acknowledge the facilities as well as the scientific and technical assistance from the NSW Node of the Australian National Fabrication Facility (ANFF) and the Research \& Prototype Foundry Core Research Facility at the University of Sydney, part of the ANFF. The authors also thank for the use of facilities at the Electron Microscope Unit within the Mark Wainwright Analytical Centre at UNSW Sydney. N. Saito is grateful to the advanced graduate course on molecular systems for devices, a program for leading graduate schools, Kyushu University, Japan.

\section{REFERENCES}

1. Yaghi, O. M.; O'Keeffe, M.; Ockwig, N. W.; Chae, H. K.; Eddaoudi, M.; Kim, J., Reticular synthesis and the design of new materials. Nature 2003, 423, 705-714.

2. Furukawa, H.; Cordova, K. E.; O'Keeffe, M.; Yaghi, O. M., The chemistry and applications of metalorganic frameworks. Science 2013, 341, 1230444.

3. Lim, G. J. H.; Wu, Y.; Shah, B. B.; Koh, J. J.; Liu, C. K.; Zhao, D.; Cheetham, A. K.; Wang, J.; Ding, J., 3D-Printing of Pure Metal-Organic Framework Monoliths. ACS Materials Lett. 2019, 1, 147-153.

4. Chen, E. X.; Yang, H.; Zhang, J., Zeolitic imidazolate framework as formaldehyde gas sensor. Inorg. Chem. 2014, 53, 5411-5413.

5. Campbell, M. G.; Liu, S. F.; Swager, T. M.; Dinca, M., Chemiresistive Sensor Arrays from Conductive 2D Metal-Organic Frameworks. J. Am. Chem. Soc. 2015, 137, 13780-13783.

6. Trickett, C. A.; Osborn Popp, T. M.; Su, J.; Yan, C.; Weisberg, J.; Huq, A.; Urban, P.; Jiang, J.; Kalmutzki, M. J.; Liu, Q.; Baek, J.; Head-Gordon, M. P.; Somorjai, G. A.; Reimer, J. A.; Yaghi, O. M., Identification of the strong Bronsted acid site in a metal-organic framework solid acid catalyst. Nat. Chem. 2019, 11, 170-176.

7. $\quad$ Choi, K. M.; Jeong, H. M.; Park, J. H.; Zhang, Y.-B.; Kang, J. K.; Yaghi, O. M., Supercapacitors of Nanocrystalline Metal - Organic Frameworks. ACS Nano 2014, 8, 7451-7457.

8. $\quad$ Li, M.; Wan, Y.; Huang, J.-K.; Assen, A. H.; Hsiung, C.-E.; Jiang, H.; Han, Y.; Eddaoudi, M.; Lai, Z.; Ming, J.; Li, L.-J., Metal-Organic Framework-Based Separators for Enhancing Li-S Battery Stability: Mechanism of Mitigating Polysulfide Diffusion. ACS Energy Lett. 2017, 2, 2362-2367.

9. Huang, J. K.; Li, M.; Wan, Y.; Dey, S.; Ostwal, M.; Zhang, D.; Yang, C. W.; Su, C. J.; Jeng, U. S.; Ming, J.; Amassian, A.; Lai, Z.; Han, Y.; Li, S.; Li, L. J., Functional Two-Dimensional Coordination Polymeric Layer as a Charge Barrier in Li-S Batteries. ACS Nano 2018, 12, 836-843.

10. Moribe, S.; Chen, Z.; Alayoglu, S.; Syed, Z. H.; Islamoglu, T.; Farha, O. K., Ammonia Capture within Isoreticular Metal-Organic Frameworks with Rod Secondary Building Units. ACS Materials Lett. 2019 , 1, $476-480$.

11. Stassen, I.; Burtch, N.; Talin, A.; Falcaro, P.; Allendorf, M.; Ameloot, R., An updated roadmap for the integration of metal-organic frameworks with electronic devices and chemical sensors. Chem. Soc. Rev. 2017, 46, 3185-3241.

12. Shekhah, O.; Wang, H.; Zacher, D.; Fischer, R. A.; Woll, C., Growth mechanism of metal-organic frameworks: insights into the nucleation by employing a step-by-step route. Angew. Chem., Int. Ed. 2009, 48, 50385041 .

13. Virmani, E.; Rotter, J. M.; Mahringer, A.; von Zons, T.; Godt, A.; Bein, T.; Wuttke, S.; Medina, D. D., OnSurface Synthesis of Highly Oriented Thin Metal-Organic Framework Films through Vapor-Assisted Conversion. $J$. Am. Chem. Soc. 2018, 140, 4812-4819.

14. Mintova, S.; Bein, T., Microporous Films Prepared by Spin-Coating Stable Colloidal Suspensions of Zeolites. Adv. Mater. 2001, 13, 1880-1883.

15. Chernikova, V.; Shekhah, O.; Eddaoudi, M., Advanced Fabrication Method for the Preparation of MOF Thin Films: Liquid-Phase Epitaxy Approach Meets Spin Coating Method. ACS Appl. Mater. Interfaces 2016, 8 , 20459-20464. 
16. Martin, P. M., Handbook of deposition technologies for films and coatings: science, applications and technology (3rd Edition). William Andrew: Boston, 2010.

17. Stassen, I.; Styles, M.; Grenci, G.; Gorp, H. V.; Vanderlinden, W.; Feyter, S. D.; Falcaro, P.; Vos, D. D.; Vereecken, P.; Ameloot, R., Chemical vapour deposition of zeolitic imidazolate framework thin films. Nat. Mater. 2016, 15, 304-310.

18. Lausund, K. B.; Nilsen, O., All-gas-phase synthesis of UiO-66 through modulated atomic layer deposition. Nat. Commun. 2016, 7, 13578.

19. Salmi, L. D.; Heikkilä, M. J.; Puukilainen, E.; Sajavaara, T.; Grosso, D.; Ritala, M., Studies on atomic layer deposition of MOF-5 thin films. Microporous Mesoporous Mater. 2013, 182, 147-154.

20. Leskela, M.; Ritala, M., Atomic layer deposition chemistry: recent developments and future challenges. Angew. Chem., Int. Ed. 2003, 42, 5548-5554.

21. Papadopoulos, N.; Illekova, E.; Karayanni, H.; Hristoforou, E., Synthesis and characterization of cobalt precursors for the growth of magnetic thin films by the MOCVD method. J. Optoelectron. Adv. M. 2008, 10, 10981102.

22. Banerjee, R.; Phan, A.; Wang, B.; Knobler, C.; Furukawa, H.; O'Keeffe, M.; Yaghi, O. M., Highthroughput synthesis of zeolitic imidazolate frameworks and application to CO2 capture. Science 2008, 319, 939943.

23. Guo, W.; Liu, J.; Weidler, P. G.; Liu, J.; Neumann, T.; Danilov, D.; Wenzel, W.; Feldmann, C.; Wöll, C., Loading of ionic compounds into metal-organic frameworks: a joint theoretical and experimental study for the case of La3+. Phys. Chem. Chem. Phys. 2014, 16, 17918-17923.

24. Li, X.; Gao, X.; Ai, L.; Jiang, J., Mechanistic insight into the interaction and adsorption of Cr (VI) with zeolitic imidazolate framework-67 microcrystals from aqueous solution. Chem. Eng. J. 2015, 274, $238-246$.

25. Hu, Y.; Kazemian, H.; Rohani, S.; Huang, Y.; Song, Y., In situ High Pressure Study of ZIF-8 by FTIR Spectroscopy. Chem. Commun. 2011, 47, 12694-12696.

26. Dou, S.; Dong, C.-L.; Hu, Z.; Huang, Y.-C.; Chen, J.-1.; Tao, L.; Yan, D.; Chen, D.; Shen, S.; Chou, S.; Wang, S., Atomic-Scale CoOx Species in Metal-Organic Frameworks for Oxygen Evolution Reaction. Adv. Funct. Mater. 2017, 27, 1702546.

27. Gonzalez, M. I.; Turkiewicz, A. B.; Darago, L. E.; Oktawiec, J.; Bustillo, K.; Grandjean, F.; Long, G. J.; Long, J. R., Confinement of atomically defined metal halide sheets in a metal-organic framework. Nature 2020, 577, 64-68.

28. Towsif Abtab, S. M.; Alezi, D.; Bhatt, P. M.; Shkurenko, A.; Belmabkhout, Y.; Aggarwal, H.; Weseliński, Ł. J.; Alsadun, N.; Samin, U.; Hedhili, M. N.; Eddaoudi, M., Reticular Chemistry in Action: A Hydrolytically Stable MOF Capturing Twice Its Weight in Adsorbed Water. Chem 2018, 4, 94-105.

29. Li, M.; Zhao, Z.; Cheng, T.; Fortunelli, A.; Chen, C. Y.; Yu, R.; Zhang, Q.; Gu, L.; Merinov, B. V.; Lin, Z.; Zhu, E.; Yu, T.; Jia, Q.; Guo, J.; Zhang, L.; Goddard, W. A., 3rd; Huang, Y.; Duan, X., Ultrafine jagged platinum nanowires enable ultrahigh mass activity for the oxygen reduction reaction. Science 2016, 354, 1414-1419.

30. Schoedel, A.; Scherb, C.; Bein, T., Oriented Nanoscale Films of Metal-Organic Frameworks By RoomTemperature Gel-Layer Synthesis. Angew. Chem., Int. Ed. 2010, 49, 7225-7228.

31. Nakamoto, K.; McCarthy, P. J.; Martell, A. E., Infrared Spectra of Metal Chelate Compounds. III. Infrared Spectra of Acetylacetonates of Divalent Metals1. J. Am. Chem. Soc. 1961, 83, 1272-1276.

32. Xia, W.; Zhu, J.; Guo, W.; An, L.; Xia, D.; Zou, R., Well-defined carbon polyhedrons prepared from nano metal-organic frameworks for oxygen reduction. J. Mater. Chem. A 2014, 2, 11606-11613.

33. Morris, R. E.; James, S. L., Solventless synthesis of zeolites. Angew. Chem., Int. Ed. 2013, 52, $2163-2165$.

34. Yoon, M.; Suh, K.; Natarajan, S.; Kim, K., Proton conduction in metal-organic frameworks and related modularly built porous solids. Angew. Chem., Int. Ed. 2013, 52, 2688-2700.

35. Song, Z.; Huang, Y.; Xu, W. L.; Wang, L.; Bao, Y.; Li, S.; Yu, M., Continuously Adjustable, MolecularSieving "Gate" on 5A Zeolite for Distinguishing Small Organic Molecules by Size. Sci. Rep. 2015, 5, 13981.

36. Kentish, S. E.; Scholes, C. A.; Stevens, G. W., Carbon dioxide separation through polymeric membrane systems for flue gas applications. Recent Pat. Chem. Eng. 2008, 1, 52-66.

37. Borjigin, T.; Sun, F.; Zhang, J.; Cai, K.; Ren, H.; Zhu, G., A microporous metal-organic framework with high stability for GC separation of alcohols from water. Chem. Commun. 2012, 48, 7613-7615.

38. Somorjai, G. A.; Li, Y., Introduction to surface chemistry and catalysis. John Wiley \& Sons: 2010. 
a

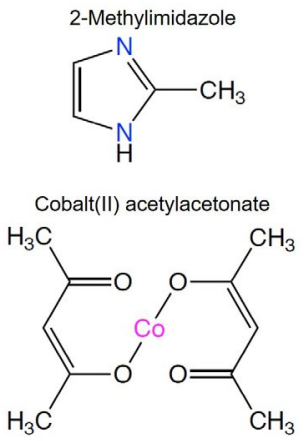

c

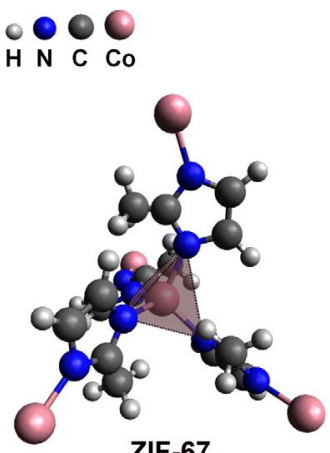

b
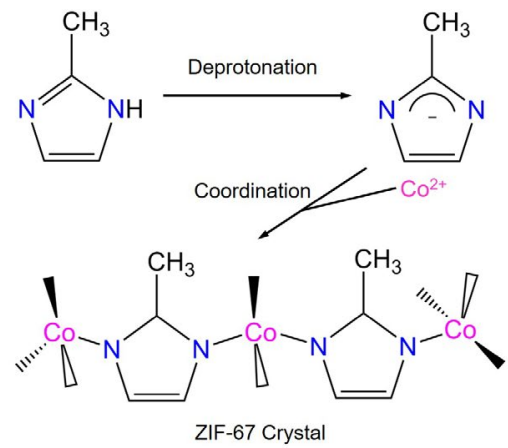

d

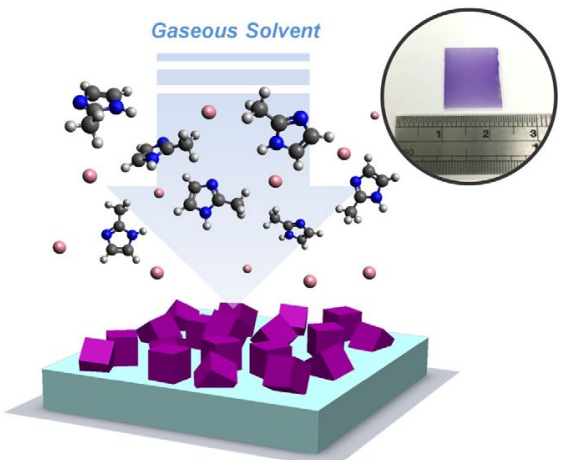

Figure 1. Steam-assisted CVD synthesis of porous ZIF-67. (a) 2-Methylimidazole and Cobalt(II) acetylacetonate serve as the precursors in the CVD process. (b) Formation pathway of the growth of ZIF-67 crystals. (c) The tetrahedral unit of ZIF-67 is presented to illustrate the coordination environment of cobalt atoms. (d) Schematic illustration for the process of steamassisted chemical vapor deposition of ZIF-67. The inset shows the photograph of a uniform ZIF67 thin film grown on a c-sapphire substrate. 

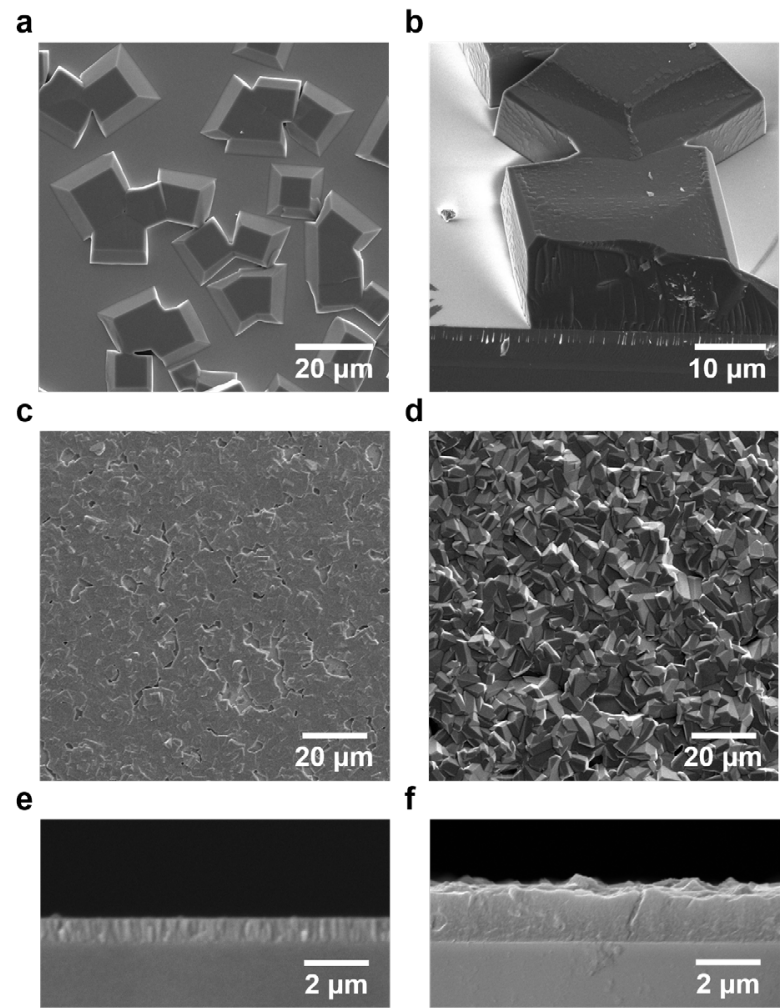

Figure 2. Morphological characterizations of CVD ZIF-67. (a) SEM image of the as-grown ZIF-67 on a c-sapphire substrate. (b) 45 degree tilted SEM image of the as-grown ZIF-67 crystals. Top-view and cross-section SEM images of compact ZIF-67 thin film grown on a csapphire substrate under $(c, e)$ low growth rate and $(d, f)$ high growth rate, respectively. 
a

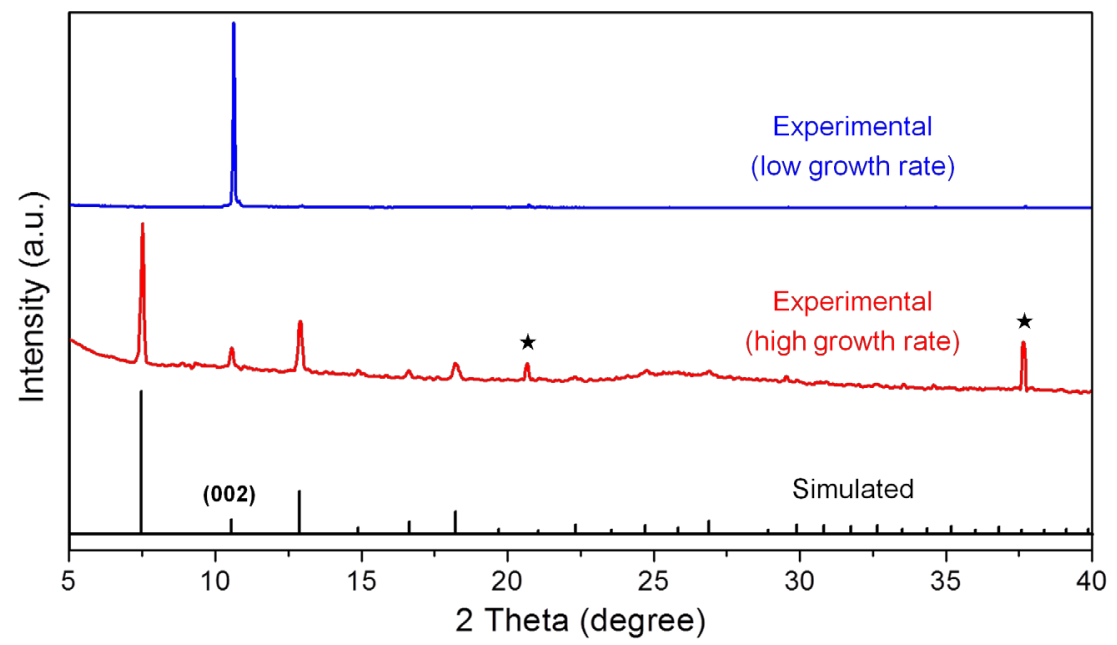

b

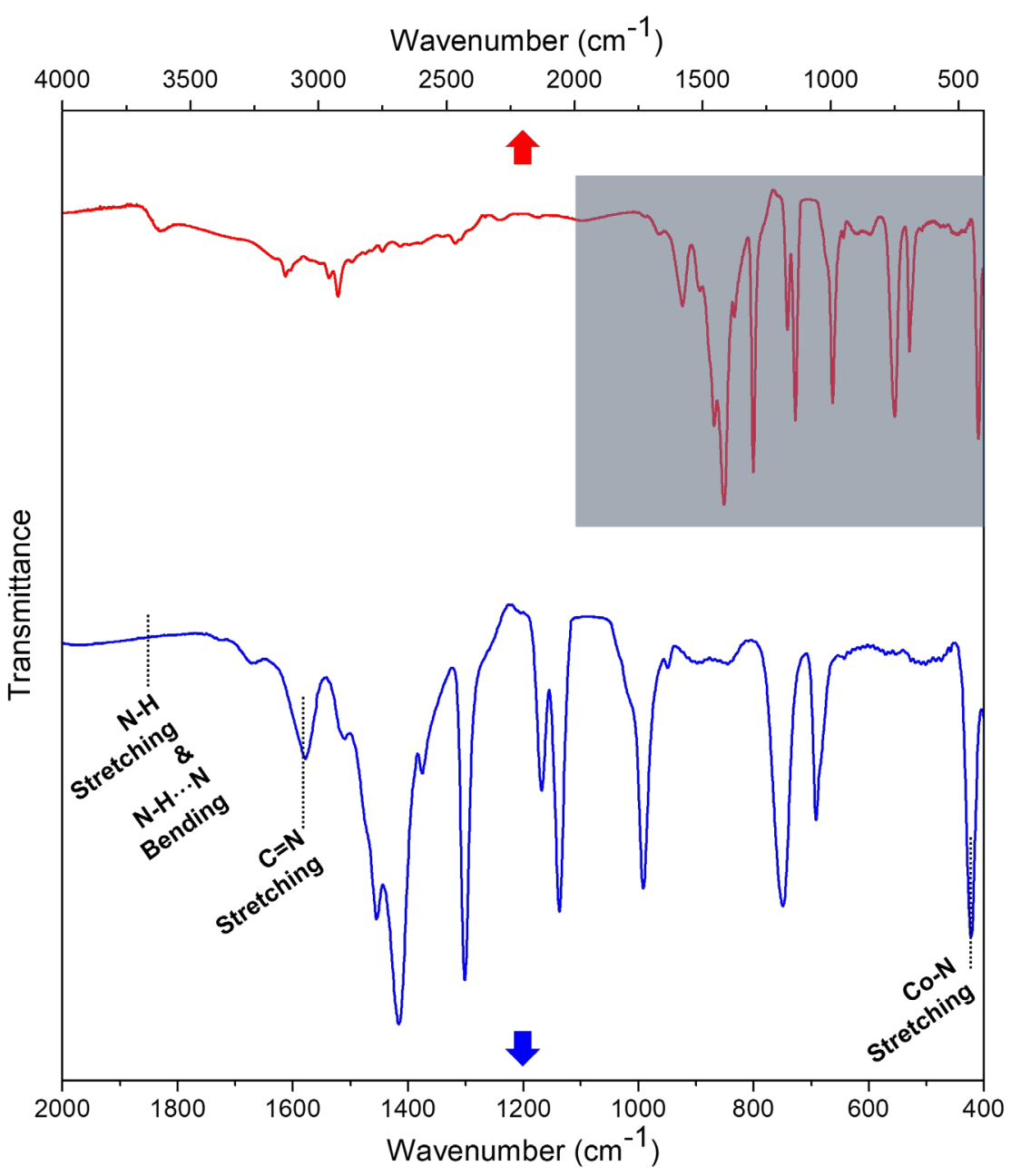

Figure 3. Spectroscopic characterization of CVD ZIF-67. (a) The X-ray diffraction pattern of an as-grown ZIF-67 thin film with different growth rates (red \& blue) and simulated pattern for ZIF-67 (black). Star marks represent the peaks from the c-sapphire substrate. (b) Full FTIR spectrum (red) of CVD ZIF-67 and corresponding zoom-in fingerprint region (blue). 
a

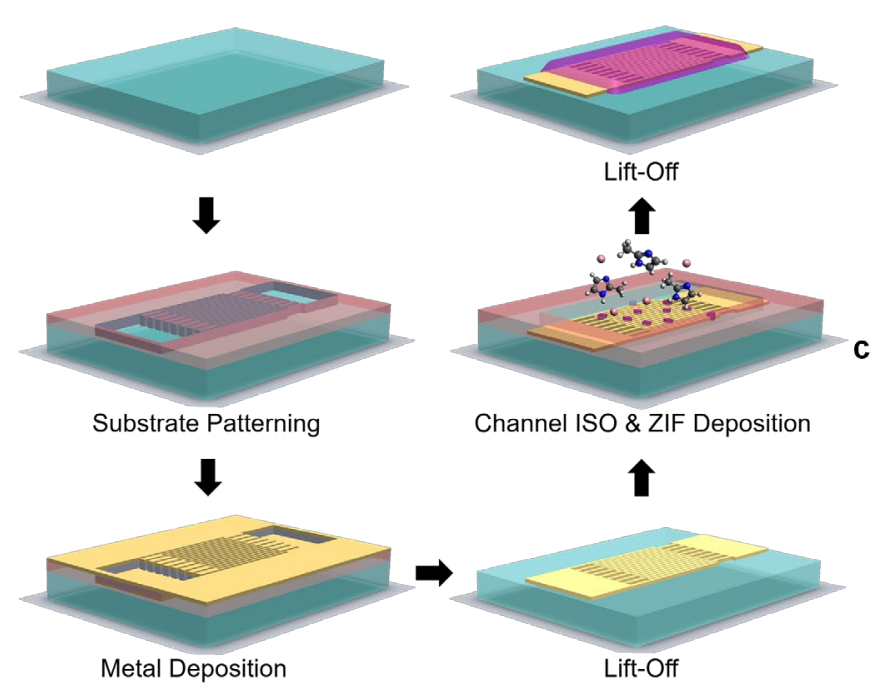

b

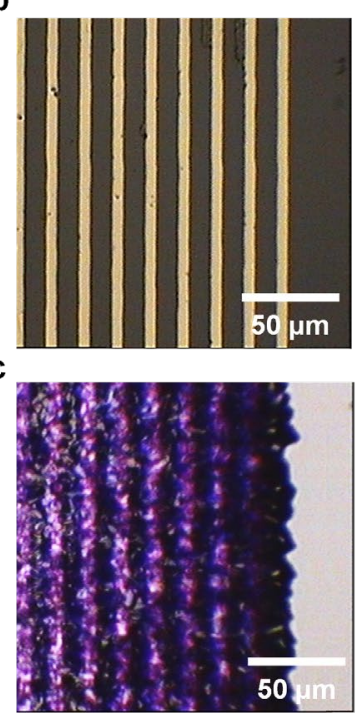

Figure 4. ZIF-67 chemiresistor fabrication. (a) Schematic illustration of the preparation of ZIF67 chemiresistor. The optical image of interdigital electrodes (b) before ZIF-67 depositing and (c) after ZIF-67 depositing. 

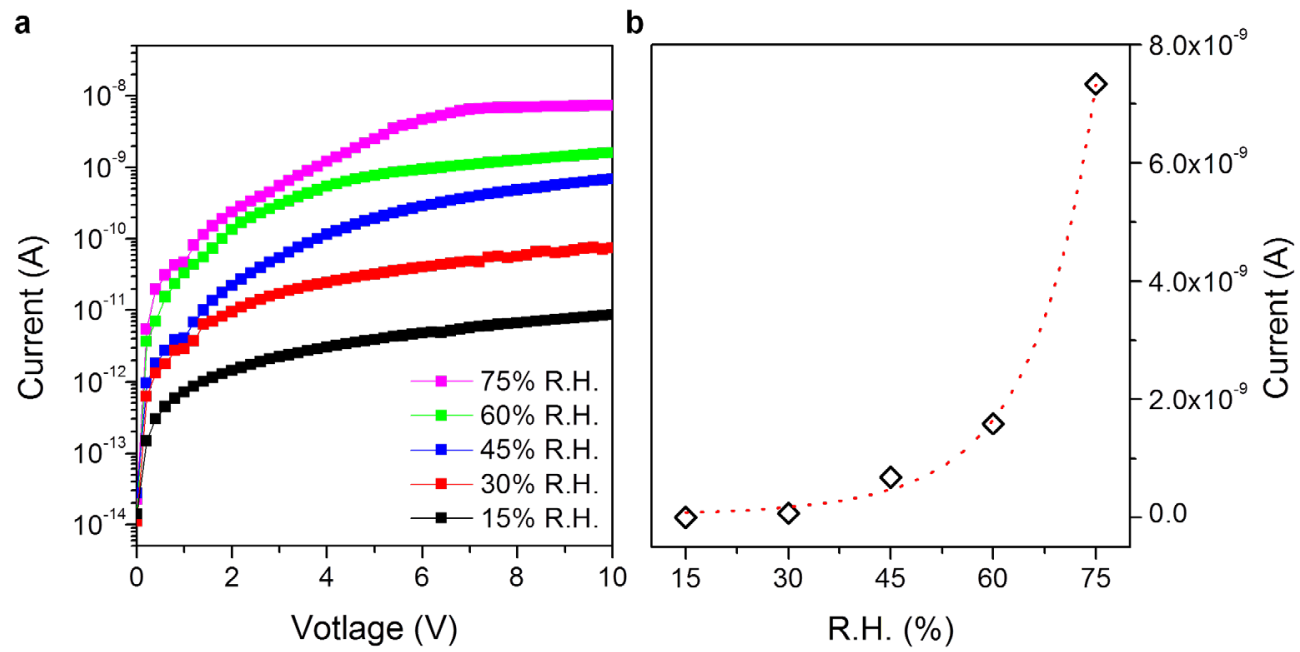

C

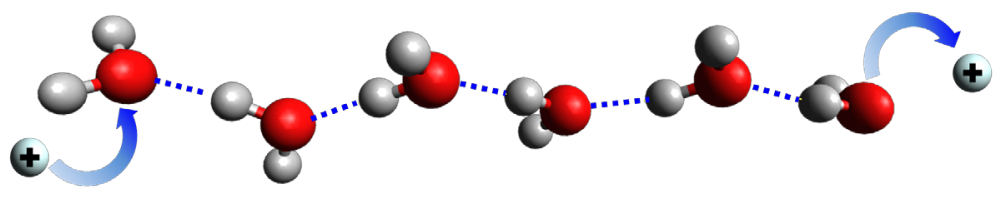

d

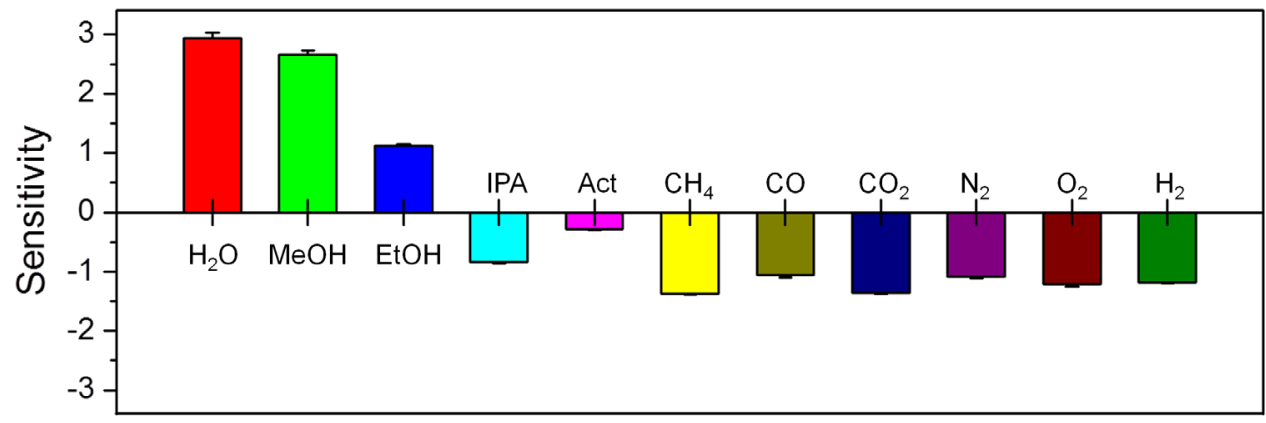

Figure 5. Chemiresistive gas sensor enabled by CVD ZIF-67. (a) Output characteristics of the CVD ZIF-67 chemiresistor at various humidity conditions. (b) Dependence of current on the relative humidity of the chemiresistor obtained from CVD ZIF-67 at room temperature and 10V. The dots represent the measured data; the red dashed line shows an exponential fit for interpolation. (c) Schematic illustration of protons tunneling across a series of hydrogen bonds between water molecules. (d) The sensitivity of CVD ZIF-67 chemiresistor to a variety of gases at ambient condition. 
Table of contents: A steam-aided chemical vapor deposition approach for directly producing zeolitic imidazolate frameworks (ZIFs) is demonstrated. The introduction of solvent steam, as a mediator, thoroughly prompts ZIF crystal ripening. The approach not only opens new avenues to explore various metal-organic frameworks (MOFs) preparing ways but also expedites the integration of related materials in nanofabrication.

Keyword: Chemical Vapor Deposition; Metal-Organic Framework; Chemiresistor

Title: Steam-Assisted Chemical Vapor Deposition of Zeolitic Imidazolate Framework

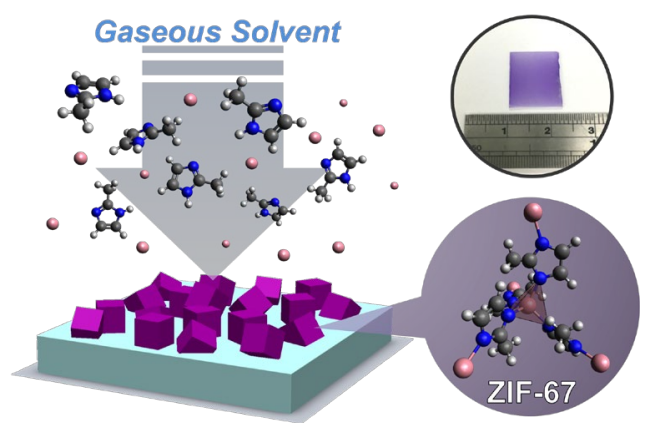

